\title{
Predicting factors of ground-glass lung nodule for growth
}

\author{
Takatoshi Aoki \\ Department of Radiology, University of Occupational and Environmental Health School of Medicine, Kitakyushu, Japan \\ Correspondence to: Takatoshi Aoki. Department of Radiology, University of Occupational and Environmental Health, 1-1 Iseigaoka, Yahatanishi-ku, \\ Kitakyushu 807-8555, Japan. Email: a-taka@med.uoeh-u.ac.jp. \\ Provenance: This is an invited Editorial commissioned by the Section Editor Dr. Jie Dai (Department of Thoracic Surgery, Shanghai Pulmonary \\ Hospital, Tongji University, Shanghai, China). \\ Comment on: Shewale JB, Nelson DB, Rice DC, et al. Natural history of ground-glass lesions among patients with previous lung cancer. Ann Thorac \\ Surg 2018;105:1671-7.
}

Submitted Aug 19, 2018. Accepted for publication Aug 27, 2018.

doi: $10.21037 /$ jtd.2018.08.129

View this article at: http://dx.doi.org/10.21037/jtd.2018.08.129

\section{Introduction}

Adenocarcinoma accounts for more than $50 \%$ of lung malignancy. Most patients with early-stage adenocarcinoma have no symptom, and a lung nodule detected at radiologic examination is common their initial finding. Small lung adenocarcinomas have been increasingly detected, and this trend is anticipated to increase due to recent advances in diagnostic technologies along with the advent of low-dose CT screening for lung cancer (1).

Ground-glass opacity (GGO) on CT is a hazy increased attenuation area of the lung but with preservation of bronchial and vascular markings, and lung nodule with GGO is named ground-glass nodule (GGN) (2). Adenocarcinomas are the most prevalent malignant lesions showing GGNs on CT. Although initial CT assessment of margins and internal characteristics are sometimes useful for a differential diagnosis of GGNs, other various lesions share some of the same adenocarcinoma CT findings. Malignant potential and identifying risk of aggressive behavior of GGNs has been attracting interest.

The revised Fleischner Society Guidelines for management of pulmonary nodules were recently published in 2017 (3). These guidelines for nodule management are based on estimations of the individual risk of malignancy. The solid nodule managements differ with size, number (single/multiple), and clinical risk factors, including age, smoking, upper lobe location, and history of lung cancer. For GGN, the managements differ with size, number (single/multiple), and internal characteristics (pure GGN or part-solid GGN), but clinical risk factors are not considered in this type (in a summary table) at this time. Although predicting factors of GGNs on CT for growth have not been largely unknown, several interesting studies which attempted to explore them were recently published.

\section{Growth predictors of GGNs}

Shewale et al. retrospectively reviewed 210 patients with a history of lung cancer and ensuing CT evidence of GGNs, and reported that 55 (26\%) patients' GGNs were stable, $131(62 \%)$ resolved, and $24(11 \%)$ progressed (4). Of the 24 GGOs that progressed, 3 were subsequently diagnosed as adenocarcinoma. On multivariable analysis, only prior adenocarcinoma emerged as a predictor of GGN progression $(\mathrm{OR}=6.9, \mathrm{P}=0.011)$, whereas history of squamous cell carcinoma or small cell carcinoma and white race were identified as predictors of GGN resolution.

According to the previous reports, the frequency with which pure GGNs increase in size or grow the solid component for many years is approximately $10-25 \%(5-9)$. Due to the slow growth of GGNs, long-term observation is needed to clarify whether they are GGNs with tendency of growth or those without growth. Cho et al. evaluated 453 GGNs (438 pure GGNs and 15 part-solid GGNs) confirmed by CT scans with a thickness of 1 to $3 \mathrm{~mm}$ and they were each followed up for a minimum of 3 years (10). Of the 453 nodules, 15 GGNs that had remained stable for the initial period of 3 years showed subsequent growth, and the frequency was $3.3 \%$ (15 of 453 ) for the nodule-based analysis. On multivariate analysis, age 65 years or older 
$(\mathrm{OR}=5.51)$, history of lung cancer $(\mathrm{OR}=6.44)$, initial size $8 \mathrm{~mm}$ or larger $(\mathrm{OR}=5.74)$, presence of a solid component $(\mathrm{OR}=16.58)$, and air bronchogram $(\mathrm{OR}=5.83)$ were independent risk factors for subsequent GGN growth.

There is another recent report attempting to link the growth of GGNs, imaging characteristics, and gene mutation. Xu et al. evaluated 69 GGNs which were followed up for 5 years to test the prediction model, and identified independent variables that could predict the volume doubling time (VDT) (11). In this study, the growing GGNs showed significantly larger initial volume on CT, maximum CT attenuation (MAX), mean CT attenuation (MEN), standard deviation of CT attenuation (STD) than the stable ones $(\mathrm{P}<0.05)$. In addition, they performed multivariate linear stepwise regressions based on the 203 independent patients with GGNs that were confirmed adenocarcinoma to calculate P53-labeling index (LI) prediction from CT measurements, and the P53-LI prediction, calculated as $0.013 \times \mathrm{MEN}+0.024 \times \mathrm{STD}+9.741$, demonstrated excellent performance in predicting growth of GGNs (AUC $=0.833, \mathrm{P}<0.001)$, and independently forecasted VDT of GGNs $(\beta$-coefficients $=1.773, \mathrm{P}<0.001)$. The authors concluded that the P53-LI prediction that was calculated from quantitative CT measurements of GGNs allows for predicting growth of GGNs.

\section{Summary and comments}

In recent years, predicting factors of GGNs for growth has been gradually revealed. A number of researchers have attempted to correlate CT findings with pathological prognostic factors and survival in adenocarcinomas showing GGNs and have reported that the adenocarcinomas GGO component can be a good predictor of lymph node metastasis and larger GGO component in adenocarcinoma correlates with favorable outcome (12-16). However, predicting growth of GGNs seems not to be as simple as them.

Because of more indolent growth for cancerous GGNs, longer initial follow-up intervals and longer total follow-up periods are recommended for GGNs than for solid nodules. Meanwhile, most GGNs are stable for 3 to 4 years and nearly $1 \%$ of solitary pure GGNs $5 \mathrm{~mm}$ or smaller may develop into adenocarcinomas over many years (5). Repeated CT scans over several years has nonnegligible harms, such as anxiety, radiation exposure, and false positives results. Therefore, risk-based follow-up management for them is greatly desired.
Many more further studies from diversified standpoints are certainly needed to be able to identify factors affect the progression of GGNs and to establish the robust prediction model. Radiogenomics analysis that simultaneously attempted to link the growth of GGNs, imaging characteristics, and gene mutation, such as the aforementioned clinical study by $\mathrm{Xu}$, might be a valuable idea (17). The analysis by advanced artificial intelligence may also be a promising way to elucidate it. Consideration of suitable management that achieves the most benefit for the patients with GGNs would have to be continued.

\section{Acknowledgements}

None.

\section{Footnote}

Conflicts of Interest: The author has no conflicts of interest to declare.

\section{References}

1. Pedersen JH, Saghir Z, Wille MM, et al. Ground-glass opacity lung nodules in the era of lung cancer ct screening: radiology, pathology, and clinical management. Oncology (Williston Park) 2016;30:266-74.

2. Hansell DM, Bankier AA, MacMahon H, et al. Fleischner society: glossary of terms for thoracic imaging. Radiology 2008;246:697-722.

3. MacMahon H, Naidich DP, Goo JM, et al. Guidelines for Management of Incidental Pulmonary Nodules Detected on CT Images: From the Fleischner Society 2017. Radiology 2017;284:228-43.

4. Shewale JB, Nelson DB, Rice DC, et al. Natural History of Ground-Glass Lesions Among Patients With Previous Lung Cancer. Ann Thorac Surg 2018;105:1671-7.

5. Kakinuma R, Muramatsu Y, Kusumoto M, et al. Solitary Pure Ground-Glass Nodules $5 \mathrm{~mm}$ or Smaller: Frequency of Growth. Radiology 2015;276:873-82.

6. Chang B, Hwang JH, Choi YH, et al. Natural history of pure ground-glass opacity lung nodules detected by lowdose CT scan. Chest 2013;143:172-8.

7. Matsuguma H, Mori K, Nakahara R, et al. Characteristics of subsolid pulmonary nodules showing growth during follow-up with CT scanning. Chest 2013;143:436-43.

8. Kobayashi Y, Fukui T, Ito S, et al. How long should small lung lesions of ground-glass opacity be followed? J Thorac 
Oncol 2013;8:309-14.

9. Lee SW, Leem CS, Kim TJ, et al. The long-term course of ground-glass opacities detected on thin-section computed tomography. Respir Med 2013;107:904-10.

10. Cho J, Kim ES, Kim SJ, et al. Long-Term Follow-up of Small Pulmonary Ground-Glass Nodules Stable for 3 Years: Implications of the Proper Follow-up Period and Risk Factors for Subsequent Growth. J Thorac Oncol 2016;11:1453-9.

11. Xu X, Wu K, Zhao Y, et al. Stage I lung adenocarcinoma: the value of quantitative CT in differentiating pathological subtypes and predicting growth of subsolid nodules. Medicine (Baltimore) 2017;96:e6595.

12. Aoki T, Tomoda Y, Watanabe H, et al. Peripheral lung adenocarcinoma: correlation of thin-section CT findings with histologic prognostic factors and survival. Radiology 2001;220:803-9.

13. Matsuguma H, Yokoi K, Anraku M, et al. Proportion of ground-glass opacity on high-resolution computed

Cite this article as: Aoki T. Predicting factors of ground-glass lung nodule for growth. J Thorac Dis 2018;10(Suppl 33):S3927S3929. doi: 10.21037/jtd.2018.08.129 tomography in clinical T1 N0 M0 adenocarcinoma of the lung: A predictor of lymph node metastasis. J Thorac Cardiovasc Surg 2002;124:278-84.

14. Asamura H, Suzuki K, Watanabe S, et al. A clinicopathological study of resected subcentimeter lung cancers: a favorable prognosis for ground glass opacity lesions. Ann Thorac Surg 2003;76:1016-22.

15. Ikeda N, Maeda J, Yashima K, et al. A clinicopathological study of resected adenocarcinoma $2 \mathrm{~cm}$ or less in diameter. Ann Thorac Surg 2004;78:1011-6.

16. Suzuki K, Kusumoto M, Watanabe S, et al. Radiologic classification of small adenocarcinoma of the lung: radiologic-pathologic correlation and its prognostic impact. Ann Thorac Surg 2006;81:413-9.

17. Aoki T, Hanamiya M, Uramoto H, et al. Adenocarcinomas with predominant ground-glass opacity: correlation of morphology and molecular biomarkers. Radiology 2012;264:590-6. 